(Aus dem Laboratorium der T. Augenklinik.)

\title{
Klinische und anatomische Untersuchungen über Drusen im Sehnervenkopf.
}

\author{
Von \\ Dozenten Dr. Hans Lauber, \\ Wien. \\ Mit 43 Textabbildungen.
}

Das Augenspiegelbild der Drusen im Sehnervenkopf ist seit langer Zeit ein wohlbekanntes, so daß die Differentialdiagnose gegenüber anderen Zuständen des Sehnerven von augenärztlicher Seite wohl nur selten auf Schwierigkeiten stoßen kann. Unsere Kenntnisse über die klinische Bedeutung dieses Zustandes, seinen Einfluß auf die Funktion des Auges und seinen Zusammenhang mit anderen Augenveränderungen, sowie endlich seine Entstehungsursache bedürfen noch der weiteren Aufklärung.

Die Zahl der in der Literatur veröffentlichten Fälle ist keine übermäßig große, worauf die Ansicht beruht, daß ein einzelner kaum Gelegenheit hat viele Fälle zu sehen. Dieser Ansicht kann ich nicht ganz beipflichten, da man bei genauerer Augenspiegeluntersuchung nicht so selten einzelne Drusen im Sehnervenkopf, besonders am Rande der Sehnervenseheibe erkennen kann. Dagegen stellen Fälle mit reichlicher Entwicklung von Drusen stets Seltenheiten dar, so daß die genaue Beschreibung und Beobachtung solcher Fälle zur Lösung der strittigen Fragen beitragen kann.

Seit der großen kritischen Zusammenstellung von $\mathrm{H}_{0 \text { eg }}{ }^{47}$ ) ist noch eine Reihe von Fällen veröffentlicht worden, wodurch die Zahl der in der Literatur bekannten Fälle auf $78 \mathrm{klinisch}$ beschriebene, 21 anatomisch untersuchte beläuft, von denen 6 klinisch und anatomiseh untersucht worden sind. Es sind dies die Fälle von Hirschberg und Cirincione ${ }^{20}$ ), Gurwitseh"1), De Schweinitz ${ }^{23}$ ), Sachsalber ${ }^{28}$ ), Cirineione ${ }^{28}$ ) und Coats ${ }^{53}$ ). Diesen Fällen kann ich 14 Fälle eigener Beobachtung hinzufügen, von denen der Fall, den ich auch anatomisch zu untersuchen die Möglichkeit hatte, 1905 von Rabitsch ${ }^{41}$ ) klinisch beschrieben worden ist; weitere 7 Fälle habe ich 1907 und 1912 in der Wiener Ophthalmologischen Gesellschaft vorgestellt; die übrigen habe ich seither beobachtet. 
Im nachfolgenden werde ich zuerst die klinischen Befunde der von mir beobachteten Fälle mitteilen, sodann den Fall Rabitsch rekapitulieren und ergänzen, endlich das Ergebnis der anatomischen Untersuchung des letzteren Falles berichten. Die klinischen Fälle werde ich durch halbsehematische Zeichnungen und Gesichtsfelder erläutern, soweit sich solche in meinem Besitze befinden und wichtig erscheinen.

1. 15 jähriges Mädchen. Rechtes Auge: $\mathrm{S}=6 / 6(+1,0 \mathrm{D}$. sph.), linkes Auge: $\mathrm{S}=\% / 6\left(+0,75 \mathrm{D}\right.$. sph $+0,75 \mathrm{D}$. cyl. $\left.90^{\circ}\right)$. In beiden. Papillen größere Mengen einer leicht durchscheinenden, grauweißen, kristallinisch aussehenden Substanz,

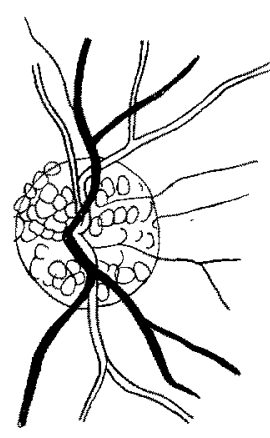

$\mathrm{Abb}, 1$.

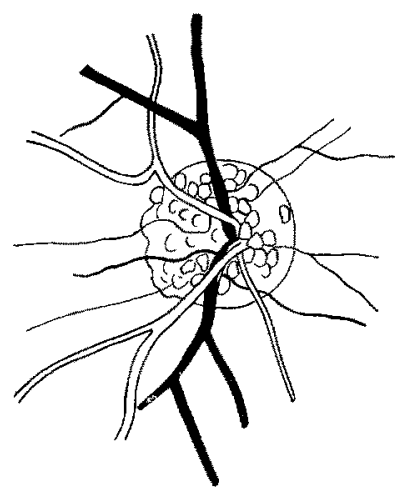

Abb. 2.

die im linken Auge die Papillengrenze leicht überschreitet und an beiden Augen eine Refraktionsdifferenz von 2,0 D. gegenüber der Netzhaut aufweist. Die Drusen verdecken die normale Struktur der Papille vollständig (Abb. 1 und 2).

2. 67 jührige Fran. Beide Angen $\mathrm{S}=6 / 6$ ohne Glas. In den Papillen beiderseits in den Randteilen krystallinische Einlagerungen, die nur wenig vorspringen.

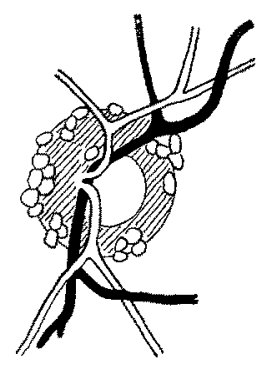

Abb. 3.

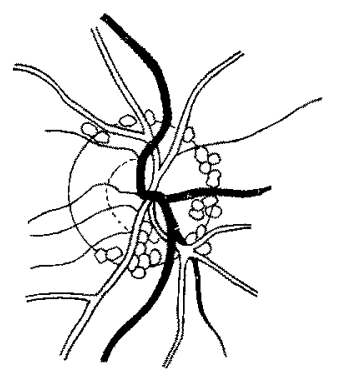

Abb. 4.

Die Begrenzung der Papille ist infolge dieser Finlagerungen etwas unregelmäßig; in der Mitte sind die seichten physiologischen Exkavationen und die Zentralgefäße deutlich sichtbar. Sonst keine Abweichung von der Norm (Abb. 3 und 4).

3. 42 jährige Klosterschwester, Tochter der vorigen. Rechtes Auge: $S=6 / 6$ $(-2,50$ D. sph. ). Linkes Auge: $\% / 6(-2,50$ D. sph.). In beiden Papillen reichliche Einlagerung derselben Substanz, welche den Ursprung der Gefäße verdeckt und bis zum Rande der Papille sich erstreckt, jedoch die rote Farbe der Papille nicht 
völlig verdeokt. Der Untersehied der Refraktion zwisehen der Kuppe oder Papille und der Netzhaut beträgt ungefähr 1,5 D. (Abb. 5 and 6).

Dieser Fall sowie der vorige und der nachfolgende wurden im Oktober 1906 untersucht und in der Ophthalmologischen Gesellschaft in Wien am 17. I. 1907 vorgestellt. Während die Mutter und die jüngere Sehwester bereits gestorben sind, ist die ältere Schwester noch am Leben und konnte am 15. I. 1921 wieder

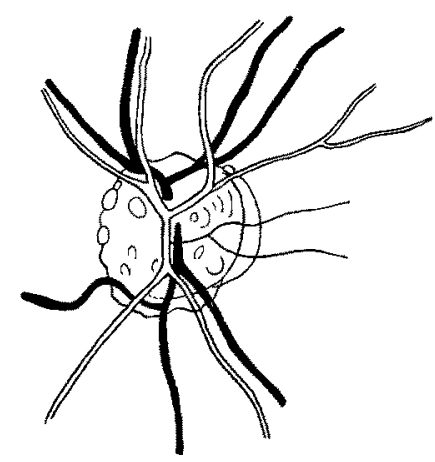

Abb. 5 .

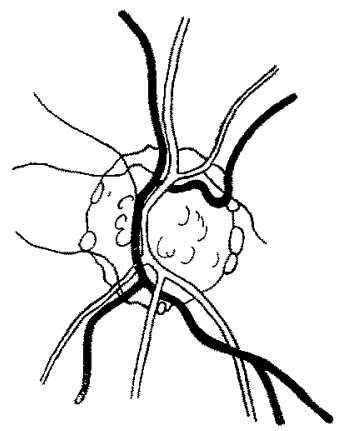

Abb. 6.

untersucht werden. Der Zustand hat sich seit 1906 merklich verändert. Die Vorwölbung der Papille hat ron 1,5 auf 3,0 D. zugenommen, die Masse der Drusen ist viel größer geworden, so daB nur in der linken Papille der naslae untere Teil noch etwas rosa erscheint; der Rest der Papille des linken Auges sowie die ganze Pa. pille des rechten Auges sind grauweiB, vollständig von Drusen verdeckt. Diese sind zum größten Teil miteinander zusammengeflossen, so daß man die einzelnen

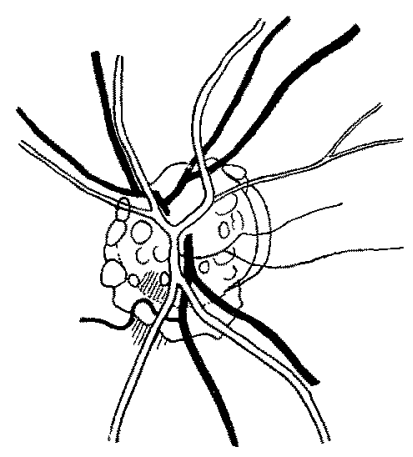

Abb. 7.

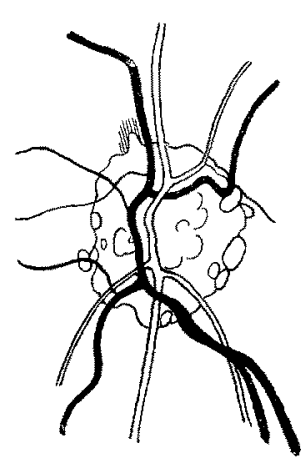

Abb. 8 .

Elemente nicht voneinander absondern kann; dies war schon 1906 der Fall gewesen. Die Funktion der Augen hat deutlich abgenommen: beiderseits ist die Sehschärfe nur noch $6 / 8$, obgleich abgesehen von den Drusen keine Veränderungen feststellbar sind. 1906 hatte man versäumt die Gesichtsfelder zu untersuchen, so daß es nicht möglich ist, sich zu vergewissern, ob diesbezüglich Veränderungen vor sich gegangen sind. Die peripheren Gesichtsfeldgrenzen für Weiß sind eingeschränkt, für Rot und Blau ist diese Einschränkung noch deutlicher ausgesprochen. Am rechten Auge, in dem die Drusenbildung einen höheren Grad erreicht hat als 
links, ist die Gesichtsfeldeinschränkung ausgesprochen; hier besteht auch nach innen oben vom blinden Fleck ein Skotom (Abb. 7-10).
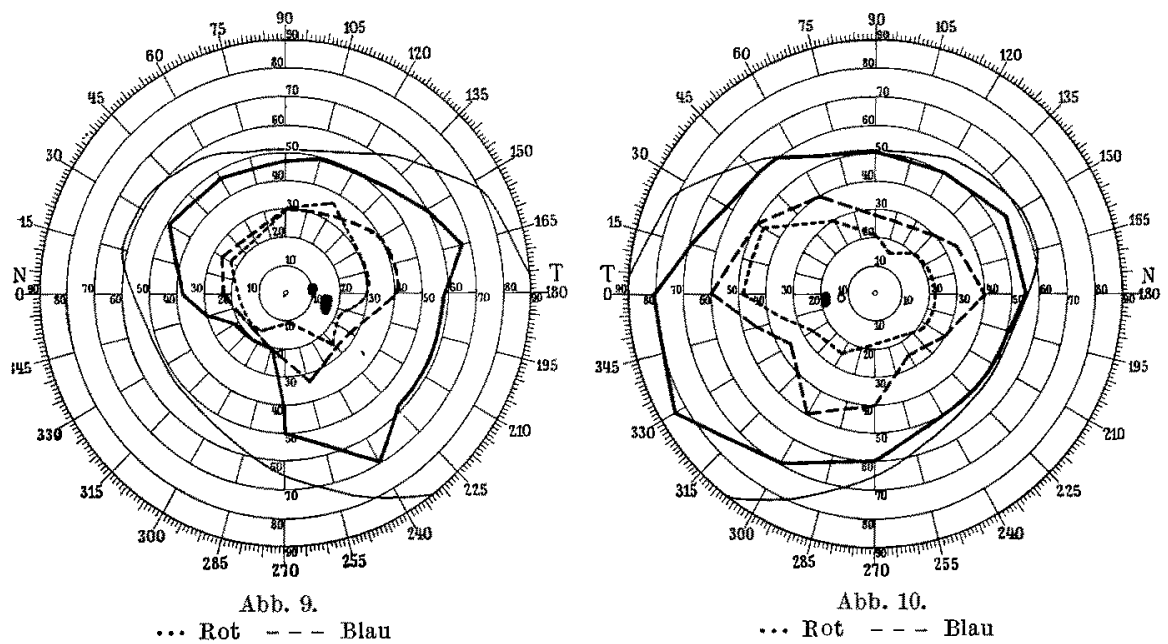

4. 41 jährige Klosterschwester, leibliche Schwester der vorigen. Beide Augen $\mathrm{S}=6 / 6(+0,25 \mathrm{~S} . \mathrm{sph}$.$) . In der Papille des rechten Auges nur geringe Andeutung$

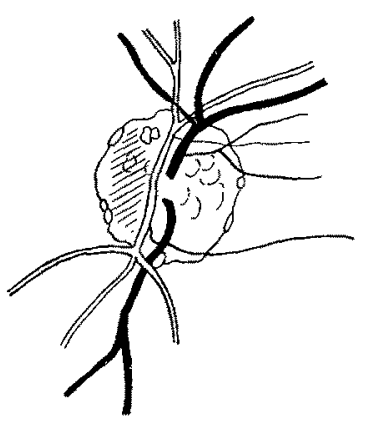

Abb. 11.

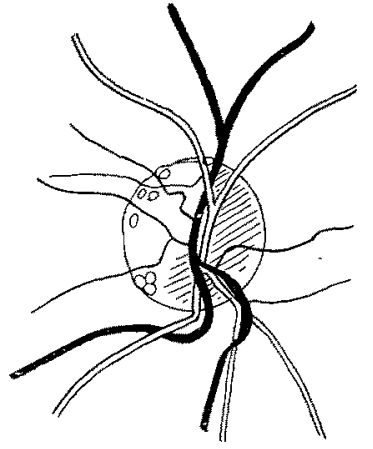

Abb. 12.

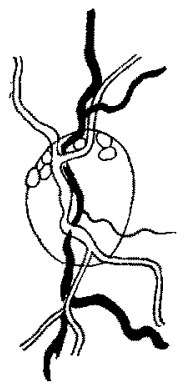

Abb. 13.

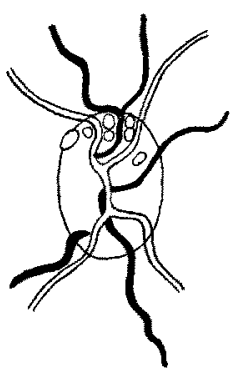

Abb. 14.

von Drusenbildung im äußeren Randteile des Sehnervenkopfes. Im linken Auge gleicht der Befund dem bei der Schwester der Kranken beiderseits bestehenden (Abb. 11 und 12).

5. 34jährige Frau. Beide Augen $\mathrm{S}=6 / 6$. Gesichtsfeld normal. Die Papillen sind leicht längsoval, etwas unscharf begrenzt, die Gefäße stark geschlängelt. Im oberen Teile der Papille finden sich beiderseits mehrere hyaline durchscheinende Schollen (Abb. 13 u. 14). 
6. 27 jähriger Mann. Beide Augen $S=6 / 6$. Im Gesichtsfeld finden sich nasal und temporal unten vom Fixationspunkte mehrere kleine Skotome. Die Papillen sind etwas unscharf begrenzt; im nasalen Teil der Papille befinden sich einige hyaline Schollen, ebenso im angrenzenden Teile der Netzhaut (Abb. 15-18).

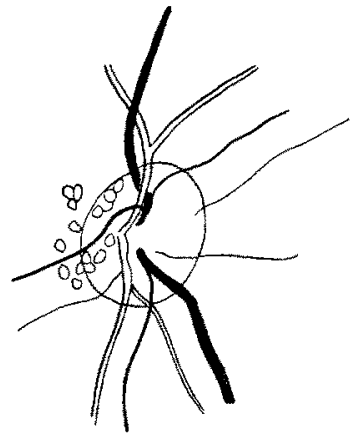

Abb. 15.

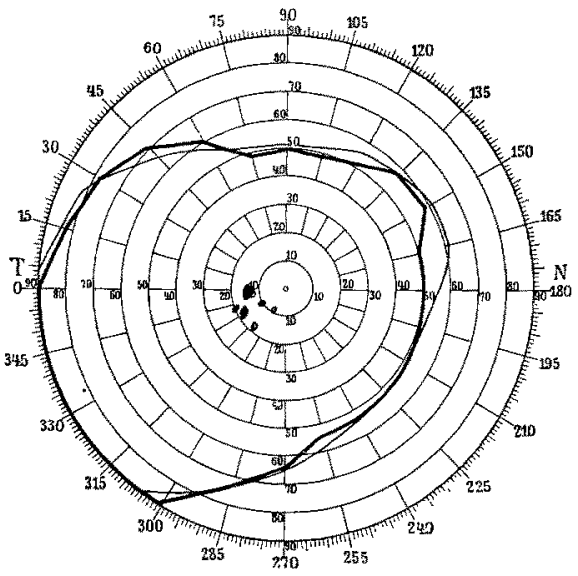

Abb. 17.

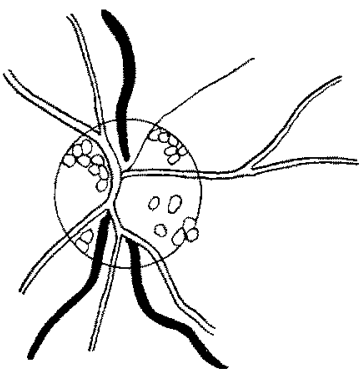

Abb. 19.

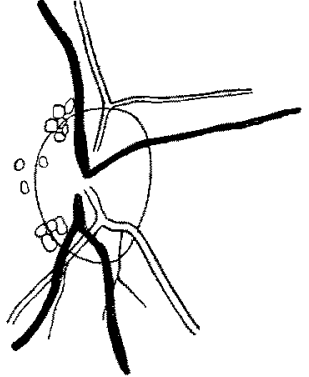

Abb. 16.

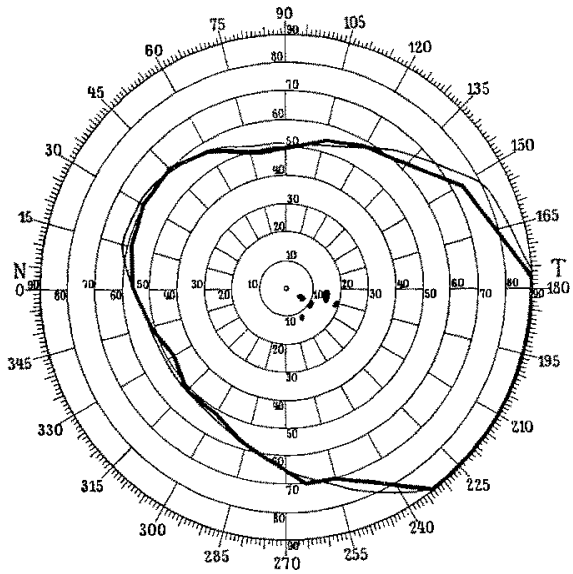

$A b b=18$.

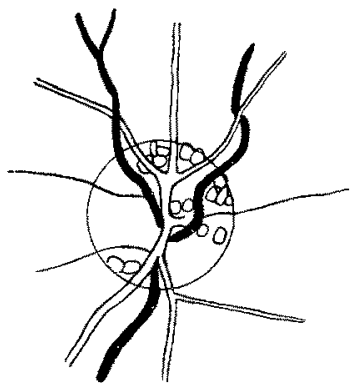

Abb. 20.

7. 30 jährige Frau. Reohtes Auge blind, linkes Auge Fingerzählen in $2 \mathrm{~m}$. Beiderseitiger Sehnervenschwund infolge eines Hypophysentumors. In beiden Papillen, besonders in der rechten zahIreiche hyaline durchscheinende Schollen. 
8. 43 jähriger Mann: Beide Augen zarte Hornhautnarben. Rechtes Auge $S=6 / 8$, linkes Auge $S=6 / 10$, fast $6 / 8^{*}$. Periphere Gesichtsfeldgrenzen normal;

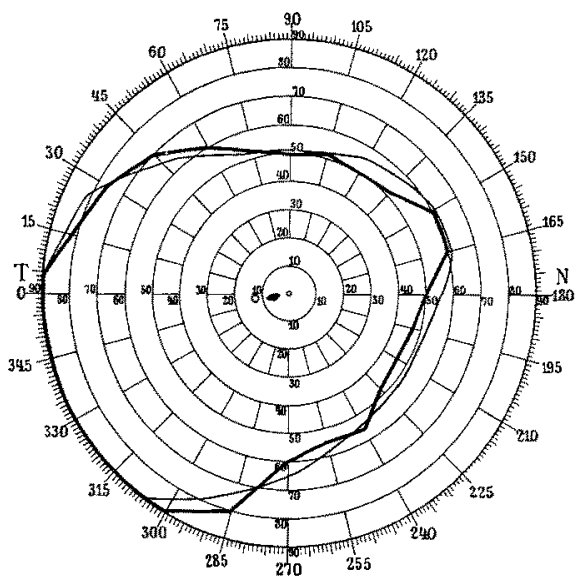

Abb. 21 . nasal vom Fixationspunkte ein kleines Strotom. In beiden Papillen. reichliche hyaline Schollen (Abb. 19 bis 21 ).

9. 50jährige Frau. Rechtes Auge $S=6 / 6(+2,25$ D. sph. $)$ linkes Auge $\mathrm{S}=6 / 10(+3,50 \mathrm{D}$. sph.). In beiden Papillen, spärliche hyaline Schollen, fast ausschließlich in der nasalen Papillenhälfte nahe dem Papillenrande. Gesichtsfeld nicht aufgenommen.

10. 56jähriger Mann. Beide Augen Fingerzählen in $1 / 2 \mathrm{~m}$. Periphere Gesichtsfeldgrenzen normal, zentrale Skotom für alle Farben außer für Gelb, relatives Skotom für WeiB. Temporale Abblassung der Papillen. Einlagerung von Drusen in geringer $\mathrm{Zahl}$ in den nasalen

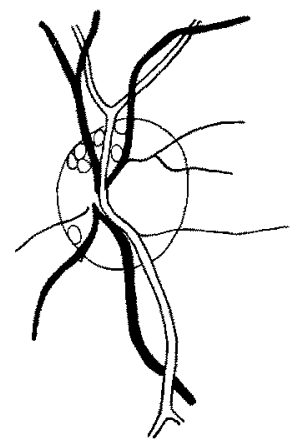

Abb. 22.

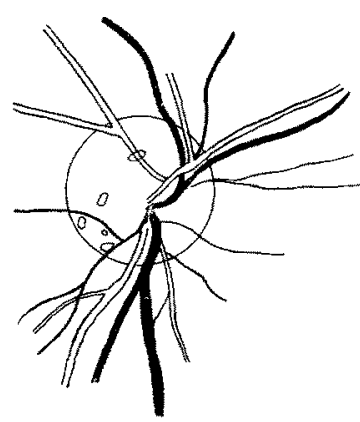

Abb. 24

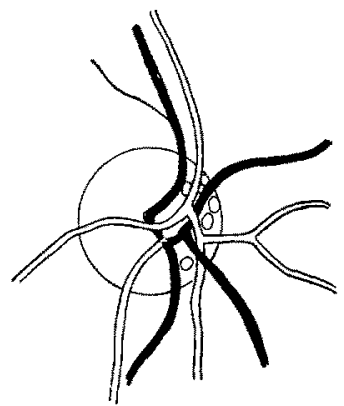

Abb. 23.

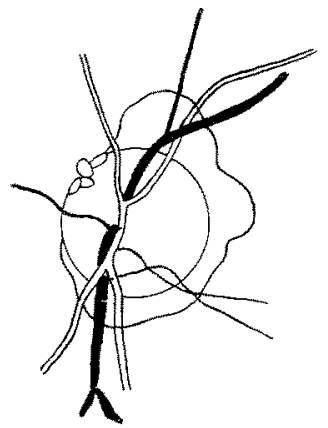

Abb. 25. Papillenhälften. Alkohol Tabak Amblyopie (Abb. 22 und 23).

\section{24jährige Medizine-} rin. Rechtes Auge $S=6 / 6$ ? $(-4,50$ D. sph. - 1,0 D. cyl. $\left.110^{\circ}\right)$. Augenhintergrund normal. Linkes Auge $\mathrm{S}=. \% \mathrm{~s}$ (-5,0 D. sph. - 1,0 D. cyl. $110^{\circ}$ ). Gesichtsfeld normal. Keine Skotome. Papille leicht anormal gebaut, indem die Exkavation stark nach der Schläfenseite der Papille verschoben ist, einen stark überhängenden inneren Rand besitzt, so daß die Gefäße ganz schräg aus ihr heraustreten. In der nasalen Papillenhälfte 5 Drusen (Abb. 24).

12. 51 jähriger Mann. Rechtes Auge $S=9 / 10(-5,0$ D. sph. $-0,75$ D. cyl. $\left.90^{\circ}\right)$. Linkes Auge ${ }^{10} / 12(=5,50 \mathrm{D}$. sph. $-1,25$ D. cyl. $90^{\circ}$ ). Beiderseits eine unregelmäBige Atrophie der Aderhaut anschließen an die Papille, dementsprechend beiderseits Vergrößerung des blinden Fleckes. Rechts periphere Gesichtsfeldgrenzen normal für Weiß und für Farben, links geringe Einschränkung 
für Farben, jedoch keine Skotome. Rechtes Auge Papille normal, linkes Auge Einlagerung von 4 Drusen in das Papillengewebe nasal oben, wobei die Drusen über den Rand der Papille etwa in die Netzhaut hineinreichen. Nach 3 Jahren Befund unverändert (Abb. 25).

13. 19 jähriger Mann. Beide Augen ${ }^{6} / 6$ ohne Korrektion. Gesichtsfeldgrenzen normal für Weiß und für Farben. Rechtes Auge keine skotome. Linkes Auge nasal unten vom Fixationspunkte zwei kleine absolute Skotome für WeiB und Farben. Beide Augen Papille scharf begrenzt, von normaler Farbe. In der Papille des rechten Auges Einlagerung von mäßig reichlichen Drusen, die vorwiegend in der inneren Hälfte der Papille liegen; Getäße von normalem Verlauf, nicht geschlängelt. Linkes Auge in der Papille etwas reichlichere Drusen als im rechten Auge; sie nehmen fast die ganze Papille ein, lassen jedoch den äußeren unteren Quadranten fast frei (Abb. 26 bis 28).

14. Fall Rabitseh ${ }^{11}$, der in den klinischen Monatsblättern für Augenheilkunde veröffentlicht worden ist. Ich habe dann den Fall 1907 in der Ophthalmologischen Gesellsehaft in Wien vorgestellt und ihn weiter bis zum Tode, der am 9. April 1914 erfolgte, verfolgen kömnen. Rabitseh macht folgende Angaben:

Th. St. wurde im Oktober 1904 zum erstenmal untersucht. Sie war damals 24 Jahre alt, stammte von gesunden Eltern; der einzige Bruder soll vollständig normale Augen haben. Sie selbst sieht schon seit früher Jugend schlecht; es wurden ihr von einem zu Rate gezogenen Arzte im 12. Lebensjahre Schutzbrillen verschrieben, $d a$ sie über Blendung klagte. Es scheint ein krankhafter Befund nicht erhoben worden zu sein. In den nächsten

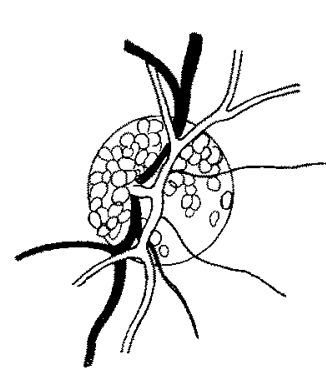

Abb. 26.

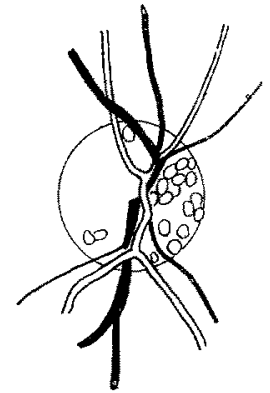

Abb. 27 .

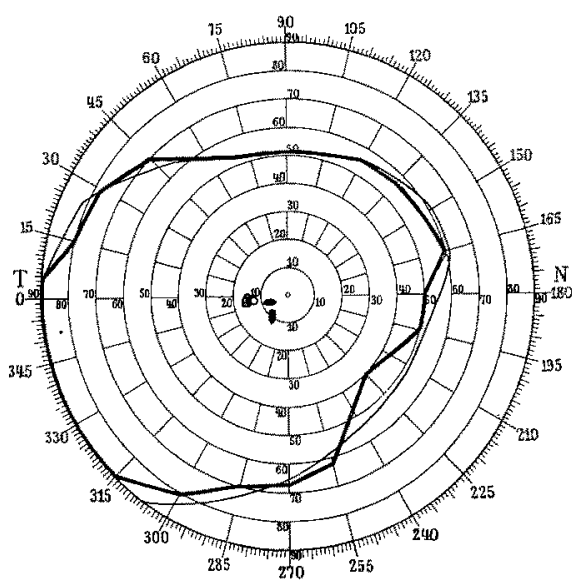

Abb. 28.

Jahren trat eine leichte Besserung ein, dann aber blieb das Sehvermögen dauernd etwas herabgesetzt. Schwankungen des Sehvermögens vorübergehender Art oder auf eine etwaige Entzündung hindeutende Anzeichen wurden niemals beobachtet. Keine Angaben über Verletzung, Hirnhautentzündung oder anderweitige Erkrankung. 1903 traten Zeichen eines Lungenleidens auf, das rasche Fortschritte machte. Die Kranke befand sich mit der Diagnose Phthisis pulmonum in Spitalsbehandlung. Seit etwa 2 Monaten fiel der Kranken auf, daB sie wohl das fixierte Objekt deutlich sehe, das übrige Gesichtsfeld war aber wie in dunklen Nebel gehüllt.

Rechtes Auge $\mathrm{S}=0,2$; im Gesichtsfeld eine unbedeutende Einschränkung. 
Linkes Auge $\mathrm{S}=0,6$, schwerer komplizierter Defekt des Gesichtsfeldes. Der Lichtsinn beiderseits mäBig herabgesetzt (Förster $9 \mathrm{~mm}$ ). (Abb. 29 und 30.)

Rechtes Auge: Die Papille erscheint vollständig deformiert, etwa trapezförmig, fast allenthalben lappig, nur stellenweise und zwar nach oben und unten unscharf begrenzt. Während sie in der Mitte annähernd den normalen rötlichen Ton besitzt, erscheint die Peripherie von gelbweißen, rundlichen Gebilden eingenommen, welche dentlich übereinander gelagert sind. Die Mehrzahl zeigt einen deutlich kugeligen Charakter, doch findet man auch solche, die dadurch, daß der Lichtreflex beständig den Rand einnimmt, fast napfförmig aussehen. Besonders schön lassen sich die einzelnen Ablagerungen bei Drehung des Spiegels voneinander sondern, wobei ihre Umrisse in wechselnden Reflexen erglänzen. Ein intensiv ,glitzerndes Körnchen in der medialen Hälfte der Papille vervollständigt das schöne Bild.

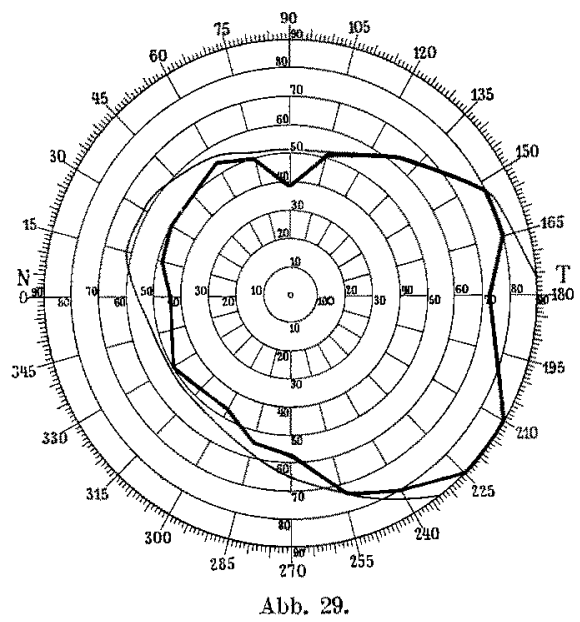

Die Netzhautgefäße liegen bedeutend über den erwähnten Drusen, weisen demgemäß diesen gegenüber eine riemlich starke parallaktische Vexschiebung auf. Die Papille macht dadurch den Eindruck pilzhutförmiger Prominenz, doch besteht nach Schnabel nur eine ganz geringe Niveaudifferenz zwischen Papillenkuppe und Netzhaut. Zwischen den Drusen und der Gefäßkuppe besteht eine Refralktionsdifferenz yon etwa 2,5 D. Die Gefäße sind in allen ihren Teilen normal, nirgends eine Wandverdickung, nirgend auch sonst im Augenbintergrund die geringsten Überreste einer etwa abgelaufenen Entzündung. Es besteht Emmetropie.

Linkes Auge: Die Verhältnisse der Papille dieses Auges unterscheiden sich nur unwesentlich von denen des rechten Auges.

Soweit die Mitteilungen von Rabitsch über diesen Fall.

Ich habe die Kranke in ständiger Beobachtung bis drei Monate vor ibren Tode gehabt. Als ich sie mit anderen Fällen von Drusen im Sehnervenkopf (Fälle 1 bis 4) am 16. Januar 1907 in der Ophthalmologischen Gesellschaft in Wien vorstellte, war der Befund noch unverändert (Abb. 31 and 32). Allmählich nahm das Sehvermögen des rechten Auges a $b$ und es bildete sich ein unregelmäßig begrenztes zentrales Skotom aus, das in seiner Gestalt nicht den. Skotomen bei retrobulbärer Neuritis glich. Die Sehschärfe war 1912 auf 0,1 (mit - 1,0 D. cyl. 180 $0^{\circ}$ gesunken. Dagegen war die Sehschärfe des linken Auges auf 1,0 gestiegen. Die periphere 
Gesichtsfeldergrenze dieses Auges war von der Nasenseite stark eingeschränkt, so dal eine nasale Hemianopsie bestand. Die schon früher vorhanden gewesenen Skotome nahmen an Größe zu. Dabei klagte die Kranke nicht über Sehstörungen, sie war vielmehr mit dem Zustande ihrer Augen zufriedener als zuvor (Abb. 33 und 34).

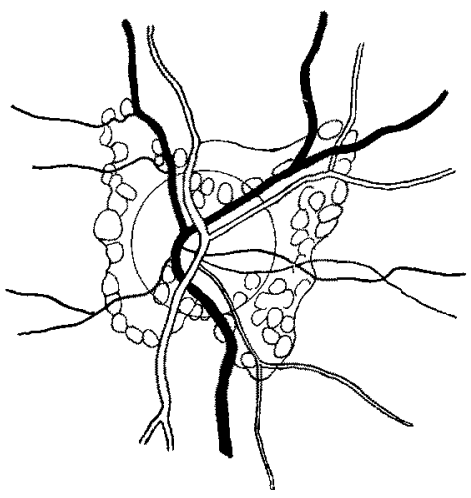

Abo. 31 .

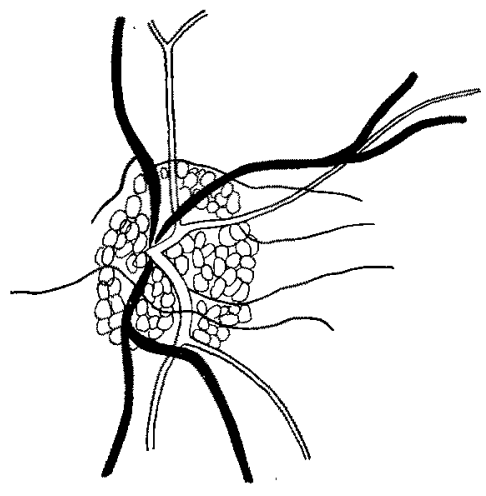

Abb. 32.

Bei der letzten genaueren Untersuchung im Dezember 1913 ergab sich folgender Befund: Rechtes Auge $3 / 60$, keine Korrektion; Gesichtsfeldgrenzen bedeutend eingeschränkt, Zentralskotom vergröBert, mit unregelmäßigen Grenzen. Linkes. Auge $\mathrm{S}=6 / 10$, Gesichtsfeld grenzen etwas enger als im vorigen Jahre, parazentrale Skotome, Gesichtsfeldmitte frei für Weiß und Farben (Abb. 35 und 36).

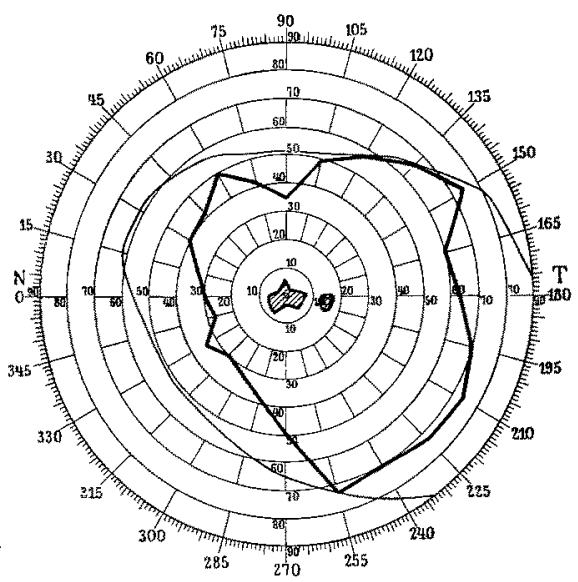

Abb. 38 .

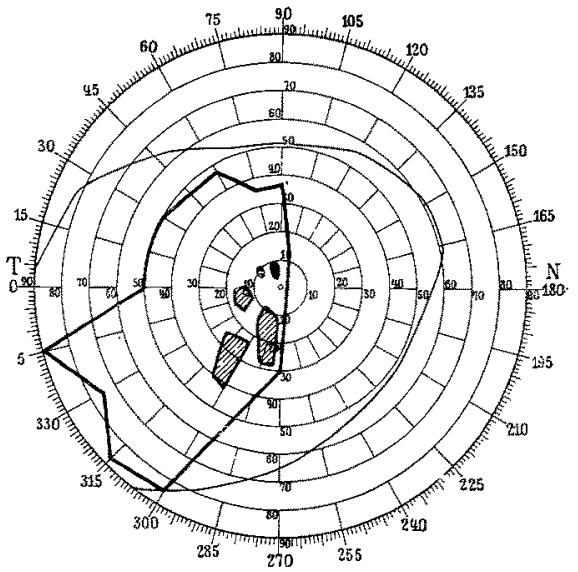

$\mathrm{Abb}, \mathbf{8 4}$.

Das Aussehen der Papillen hatte nur langsame Veränderungen erfahren. Die Zahl der Drusen hatte entschieden zugenommen und auch die Größe einzelner Körner, wie sich aus dem Vergleiche mit der Zeichnung von Rabitsch ergab, hatte zugenommen. Die Drusen verdeckten die Papille vollständig, so daß zuletzt von der rötlichen Farbe der Papille gar nichts mehr zu sehen war. Die Vorwölbung der Drusenmasse scheint zugenommen zu haben, jedoch der Unterschied in 
der Einstellung zwischen Kuppe der Papille und der Netzhautebene hatte sich fast gar nicht verändert. Der Brechungsunterschied zwischen Gefäßen und Drusenmasse, der 1904 noch 2,5 D. betragen hatte, nahm auf 1,0 D. ab (Abb. 37 und 38 ).

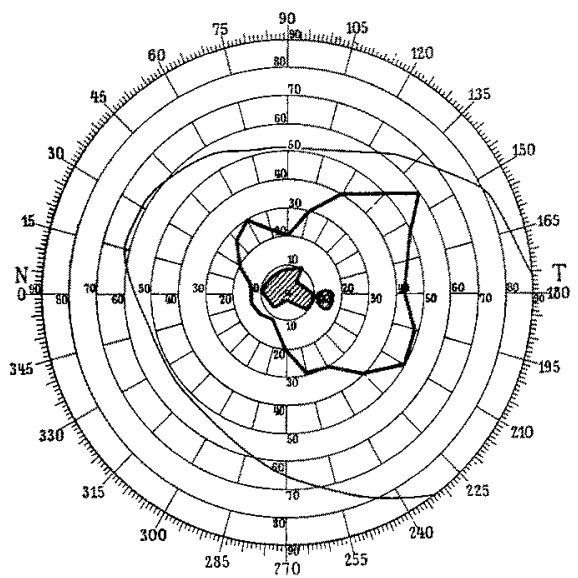

Abb. 35.

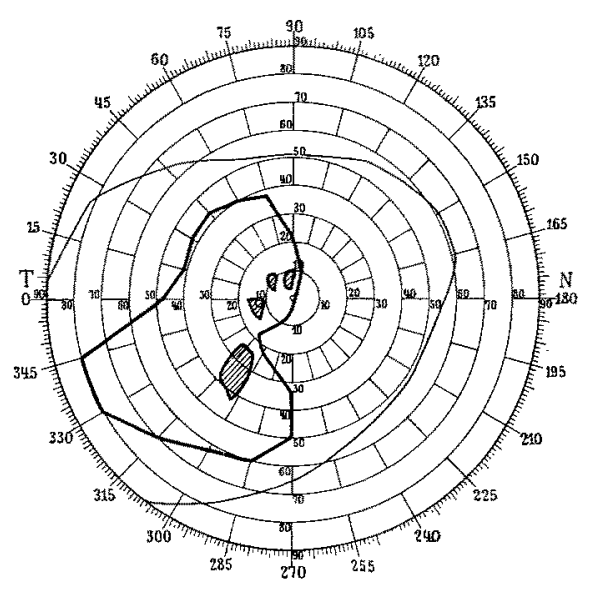

Abb. 36.

Während der Zeit nach dem Dezember 1913 war der Allgemeinzustand dex Kranken ein so schlechter, daß eine genavere Augenuntersuchung nicht vorgenommen werden konnte; es ist daher nicht sicher, ob ihr Sehvermögen während der letzten Monate ihres Lebens noch weiter abgenommen hat. In der Krankengeschichte findet sich diesbezüglich keine Angabe.

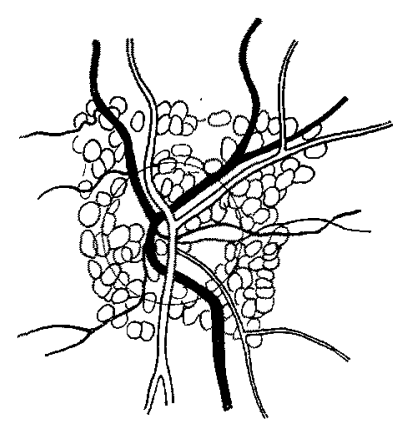

Abb. 87.

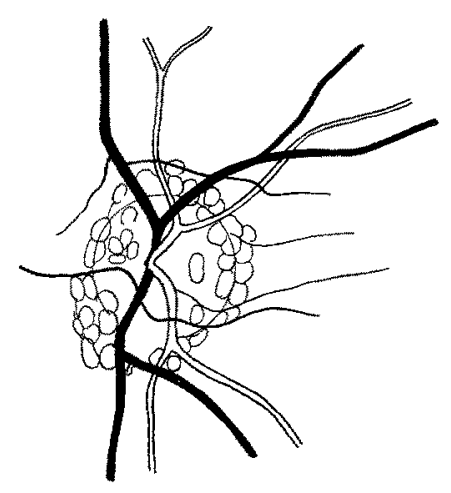

Abb. 38.

Aus dem fortschreitenden Verfall der Leistungen der Augen und der nachweis. baren Abnahme der Dicke der Schichte von Papillengewebe über den Drusen mußte der Schluß auf fortschreitenden Schwund der Nervenfasern gezogen werden, der denn auch durch die anatomische Untersuchung seine Bestätigung fand.

Die Tuberkulose, an der die Kranke seit 1903 litt, nahm immer mehr überhand; sie wurde am 19. II. 1914 auf die I. medizinische Abteilung des Jubiläumsspitales der Gemeinde Wien aufgenormmen, wo sie am 9. IV. 1914 gestorben ist. Der Obduktionsbefund lautet: Tuberculosis chronica pulmonum; tuberculosis 
ulcerosa pulmonis dextri et phthisis fibrosa pulmonis sinistri. Cystoma multiloculare ovarii dextri cum elongatione uteri et tubarum. Dilatatio varicosa vasorum lymphaticorum retroperitonealium lateris dextri. Dilatatio ureteris et pelvis renis utriusque e compressione. Atrophia nervi optici utriusque.

Das Gehirn bot keinen pathologischen Befund.

Der Freundlichkeit des Herrn Primarius Dozenten Reitter und des Herrn Prosektors Professor Maresch verdanke ich die beiden hinteren Bulbusabschnitte mit den ganzen Sehnerven einschlieBlich des Chiasma, die bei der Obduktion herausgenommen wurden. Das rechtsseitige Präparat wurde in Formol, das linksseitige mit dem Chiasma in Muller-Formol fixiert. Der Ausbruch des Krieges verhinderte mich die Präparate früher zu untersuchen und sie sind daher sehr lange in den Konservierungsflüssigkeiten gewesen, was bei der Untersuchung zu berücksichtigen war. In gewöhnlicher Weise wurden die Präparate in Celloidin eingebettet und geschnitten; dabei haben sich die Papillen ganz gut ohne vorhergehende Entkalkung schneiden lassen.

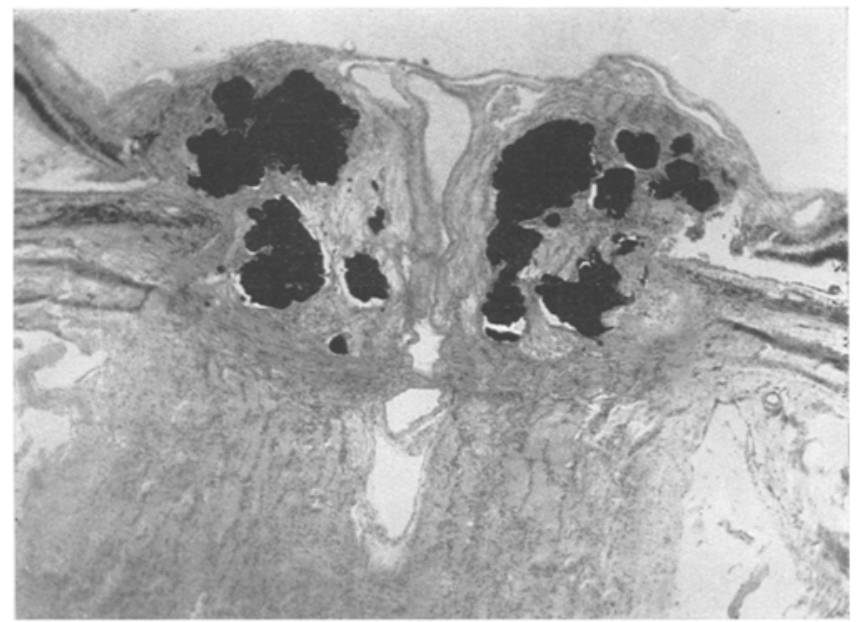

Abb. 39.

Das Ergebnis der mikroskopischen Untersuchung der beiden hinteren Angenabschnitte und der Sehnerven zeigt auf beiden Seiten annähernd dieselben Verhältnisse (Abb. 39-43).

- Die Papille springt über die Oberfläche der umgebenden Netzhaut deutlich hervor; diese Hervorragung ist durch die Einlagerung der Drusen bedingt, welche fast den ganzen Raum zwischen der Oberfläche der Papille und der Lamina cribrosa einnehmen. Die sie bedeckende Schicht von Nervenfasern ist sehr dünn, stellenweise fehlen Nervenfasern vollständig und die Drusen sind von gewucherter Glia umgeben. Die Drusen füllen über neun Zehntel des ganzen Sklerotikochorioidealkanales aus und reichen bis zu seinen Wänden, so daß die Papille ähnlich wie bei einem Glaukom ausgehöhlt erscheint, jedoch mit dem Unterschiede, daß die Exkavation von den Resten das Papillengewebes, den Gefäßen und den Drusen ausgefüllt ist. Bei der systematischen Durchmusterung der Schnitte zeigt es sich, daB die Drusenmassen zum gröBten Teil miteinander zusammenhängen, so daß sie einen fast vollständigen Ring bilden, der in der Mitte von den BlutgefäBen durchbrochen wird. Die Oberfläche der Drusenmasse ist meist eine unregelmäBig 
höckerige, doch finden sich in den großen Knollen, als welche sich die Drusen auf dem Querschnitte zeigen, buchtige Höhlungen, welche von Gliagewebe, GefäBresten und spärlichem Bindegewebe ausgefüllt sind (Abb. 42). Ganz eigentümlich ist der Befund, der an einer Stelle im Sehnerven des linken Auges er-

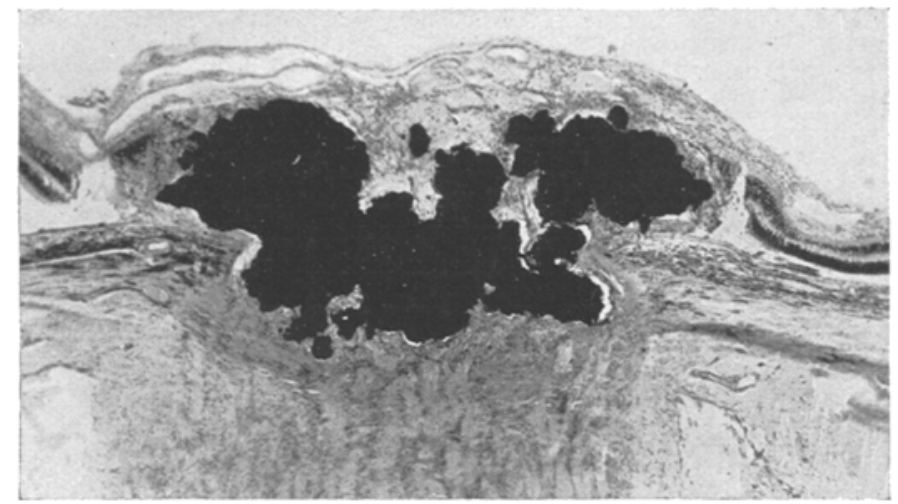

Abb. 40 .

boben wurde: mitten durch eine große Druse verläuft in einem Kanal eine kleine Vene (Abb. 43). Es zeigt sich, daß die Gefäße im Gegensatz zum Nervengewebe den Drusen Widerstand leisten, so daßB sich auch feinere Äste unversehrt erhalten, selbst wenn sie allseitig von der Drusenmasse eingeschlossen sind. Neben

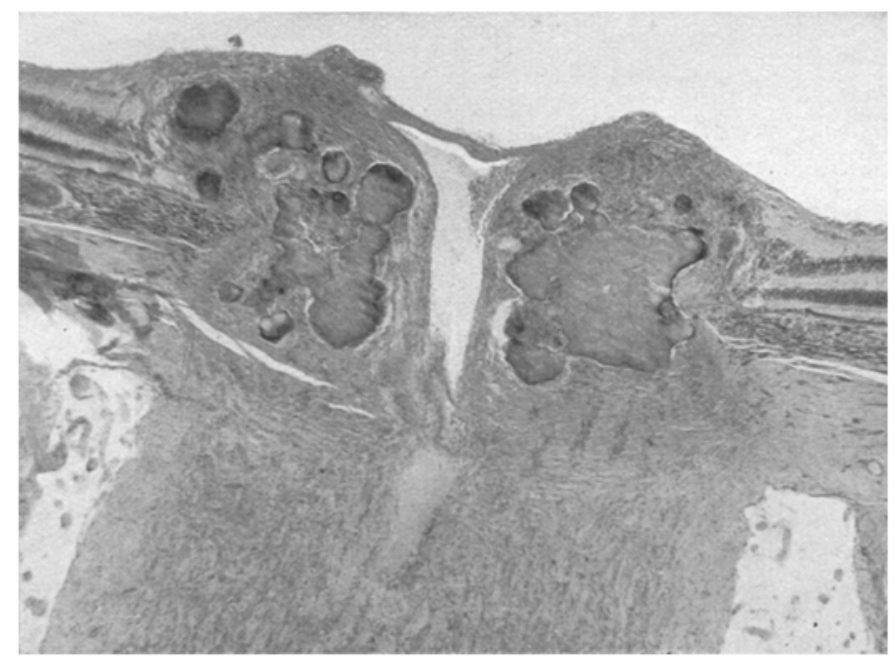

Abb. 41.

der großen Masse der zusammenhängenden Drusen liegen kleine Drusen, so dab zwischen diesen Gebilden schmale Kanäle vorhanden sind, durch die spärliche Nervenfasern verlaufen. Der Verlauf dieser Kanäle und damit der Nervenfasern ist ein unregelmäßig gewundener. Eine größere Lücke in der Drusenmasse liegt 
in der Mitte der Papille und dient den GefäBen als Durchtritt. Die Gefäße, Arterie und Vene, weisen keine Abweichungen von der Norm auf, wie sie auch klinisch keine Eigentümlichkeiten. darboten. Die Drusen liegen mitten im umgebenden Gewebe ohne bindegewebige Kapsel. Die Lamina cribrosa erscheint dichter und derber als gewöhnlich, ist im ganzen nach binten gedrängt und liegt in der Höhe der rückwärtigen Seleraloberfläche. Thr Verlauf ist leicht bogenförmiger, wie bei der glaukomatösen Exkavation. Die Drusen haben stellenweise die vordere Fläche der Lamina cribrosa usuriert und Vertiefungen in dieselbe geproßt. An einigen wenigen Stellen liegen kleine Drusen zwischen den Fasern der Lamina cribrosa, an keiner Stelle jedoch hinter ihr.

Der Sehnerv hinter der Lamina cribrosa ist stark atrophisch, bei verbreitertem Subduralraum, die Septen dicht aneinander gerïckt. Fine reaktive Vermehrung des Bindegewebes im Sehnerven ist in gleicher Weise zu verzeichnen, wie bei jeder primären Opticusatrophie es besteht, auch keine Zellvermehrung. Bei der Mark" scheidenfärbung nach PaI - Weigert zeigen sich nur einzelne leidlich gefärbte

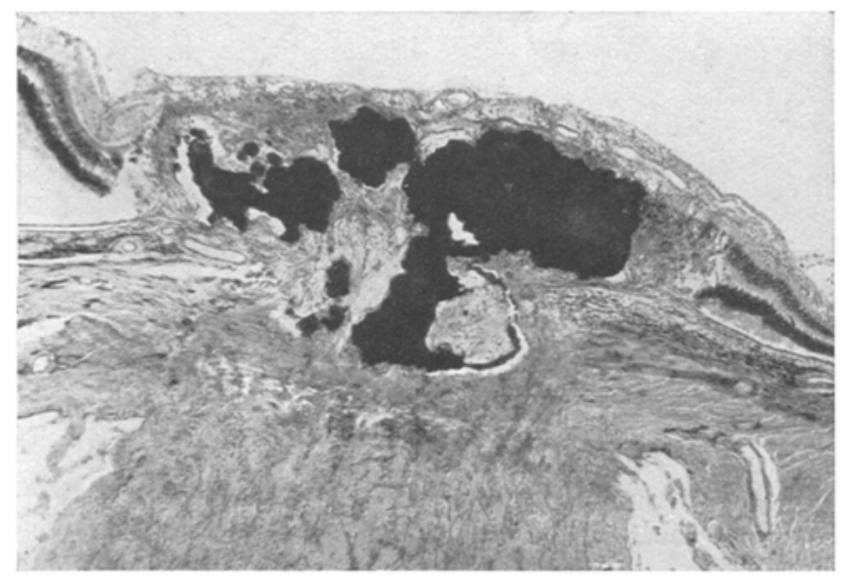

Abb. 42.

Nervenbündel. Angesichts dieses Befundes muß man annehmen, daB das Sehvermögen in der letzten Lebenszeit der Kranken noch bedeutend abgenommen habe, da eine fast vollständige Sehnervenatrophie besteht

Entsprechend dem weitgehenden Schwunde der Sehnervenfasern ist es nicht verwunderlich, daß die inneren Netzhautschichten sehr dünn sind; eine Nervenfaserschicht ist nur in Resten vorhanden, die Ganglienzellen sind spärlich und fehlen stellenweise vollständig. Die äuBeren Schichten der Netzhaut sind dagegen normal.

Dort, wo die Drusen in der Netzhaut liegen, sind die Kömerschichten auseinander gedrängt, so daß der normale Aufbau der Netzhaut nicht mehr zu erkennen ist. Das Pigmentepithel ist stellenweise geschwunden, hier und da haben sich die Pigmentzellen zusammengeballt; dort, wo die Zellen in ihrer gewöhnlichen Anordnung erhalten sind, fehlt ihr Pigment fast vollständig. An einer Stelle läßt sich erkennen, daß die Glaslamelle der Aderhaut in der Nähe des Sehnerveneintrittes fehlt; hier hat das Pigmentepithel seinen normalen Bau verloren und es ist zur Bindegewebsbildung gekommen, wobei das Bindegewebe aus der Aderhaut in die Netzhaut eingedrungen ist. Die Pigmentepithelzellen, die sich an der 
Art ihres Pigmentes erkennen lassen, sind nach vorn in die Netzhaut gewandert und finden sich auch in der unmittelbaren Umgebung einer kleinen, noch offenbar jungen Druse. Einige dieser Zellen sind gröber als normal, erscheinen gebläht und führen nur mehr Reste von Pigment; sie sind nicht unähnlich den Pigment. epithelzellen, die man oft auf der Oberfläche und in der unmittelbaren Umgebung der sog. Drusen der Glaslamelle findet. Diese selbst ist mit Ausnahme der einen erwähnten Stelle ïkerall erhalten und ragt mit dem Scleralsporn gegen die Lichtung des Sehnervenkanals vor; sie steht an keiner Stelle in Verbindung mit den Drusen. Die Aderhaut weist keine Besonderheiten auf.

Beim Vergleich der Präparate der beiden Augen fällt auf, daß die Drusen der rechten Papille (Formolfixierung) viel dunkler und deutlich blau gefärbt sind, während die Drusen in dem in Müller-Formol fixierten Präparat rötlich und viel heller erscheinen. Dies weist darauf hin, daB die Drusen ursprünglich einen größeren

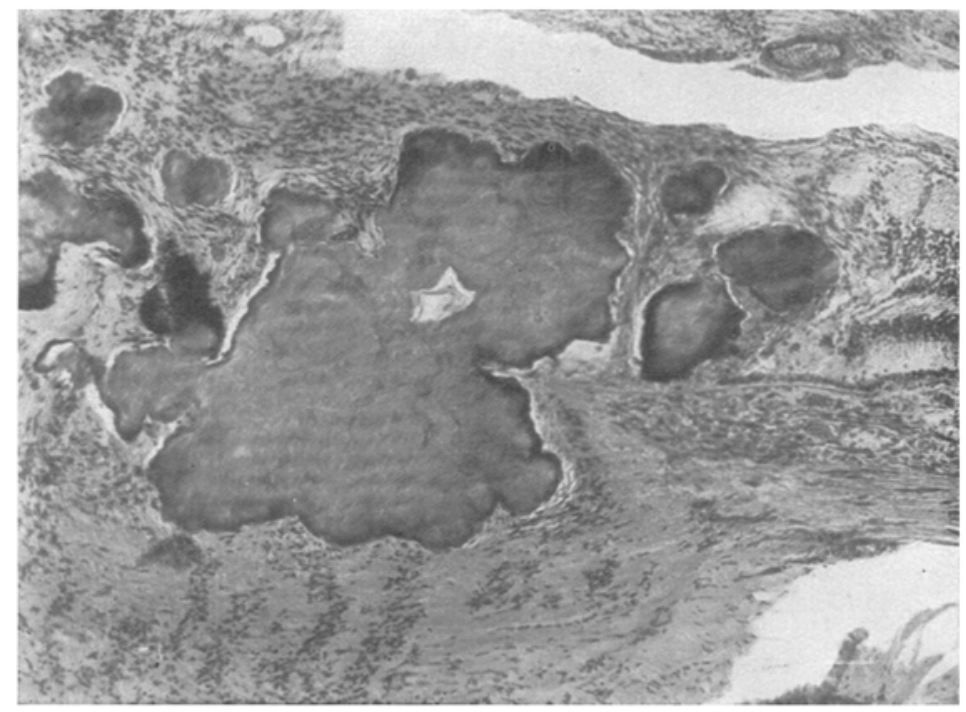

Abb. 43.

Kalkgehalt besaßen; der lange Aufenthalt in Mullerscher Flüssigkeit hat zur vollständigen Entkalkung geführt. Der Kalkgehalt war jedoch offenbar nur ein geringer, da sich das Präparat, das in Formalin gelegen war, auch ohne Entkalkung gat schneiden ließ. Diese Annahme wird durch den Umstand bestätigt, daß bei Behandlung mit Salzsäure sich im Formolpräparat Gasblasen entwickelten, deren Auftreten am anderen Präparat vermißt wurde.

An den tiefblan mit Hämatoxylin gefärbten Drusen der rechten Papille läßt sich der innere Aufbau nicht gut erkennen; er tritt dagegen in den Präparaten des anderen Auges deutlich hervor. Es zeigt sich, daß die großen Knollen aus einer großen Zahl von kleineren rundlichen Körpern bestehen, die eine Konzentrische Schichtung erkennen lassen. Diese Verhältnisse des inneren Aufbaues der Drusen sind bisher von allen Forschern beschrieben worden.

Es wurden alle Reaktionen vorgenommen, um die chemische Beschaffenheit der Drusen festzustellen. Die Drusen werden von Alkohol, Äther, Chloroform und $\mathrm{Xylol}$ nicht gelöst; ebenso bleiben Kalilauge, Ammoniak und konzentrierte 
Essigsäure ohne Wirkung. Bei Anwendung von Salzsänre oder Salpetersäure traten am Formolpräparat Gasblasen auf. Millonsches Reagens führte keine Färbung herbei. Bei Zusatz von konzentrierter Schwefelsäure trat Schwärzung und dann Auflösung zusammen mit der des ganzen Gewebes auf. Die Amyloinreaktionen (Jod-Jodkali, Jod-Jodkali mit Zusatz yon konzentrierter Sehwefelsäure, Methylviolett) fielen negativ aus mit Ausnahme der Thioninfärbung, bei der sich die Drusen hellblau färbten. Unter Einwirkung von Carminfarbstoffen nahmen die Drusen eine schwache Rosafärbung an, wobei einzelne Teile ungefärbt blieben. Säurefuchsin färbt die Drusen intensiv rot, ebenso Pikrocarmin. Weigerts Fibrinfärbung läßt die Drusen ungefärbt, bei der Russelschen Färbung nehmen sie zum großen Teil eine rote Färbung an, manche erscheinen aber auch blau. Bei der Färbung nach Mallory sind die Drusen orangegelb. Im ungefärbten Präparat haben sie einen leicht gelblichen Farbenton. Unter dem Polarisationsmilkroskope zeigen sie keine Doppelbrechung.

Eine Abweichung ron den typischen Farbreaktionen des Hyalins weist die Drusenmasse meines Falles bloß gegenüber dem Thionin auf; die Blaufärbung würde für Amyloid sprechen, wenn nicht alle anderen Amyloidreaktionen negativ ausgefallen wären.

Nach dem Ausfall all dieser Reaktionen muß man die Drusen in meinem Fall, ebenso wie in den meisten anderen anatomisch untorsuchten zum Hyalin von v. Recklinghausen zählen. Insofern bietet die vorliegende U̇ntersuchung nichts Neues. In bezug auf die Entstehung besta.tigt der vorliegende Fall die Feststellung, daB die Drusen in der Papille und der Netzhaut keine Beziehungen zur Glasmembran besitzen.

An einigen wenigen Stellen kann man in der Papille neben den Resten von Nervenfasern und gewucherter Glia große, sich mit Hämatoxylin etwas dunkler als die umgebenden Zellen färbende, geblähte Zellen finden, die stets in der unmittelbaren Nähe von kleinsten Drusen liegen. An einer Stelle ließen sich ähnliche Zellen erkennen, die, aber zweifellos vom Pigmentepithel stammen (s. oben). Es ergibt sich aus diesen Befunden die Möglichkeit anzunehmen, daß die Drusenbildung auf die Anwesenheit versprengter Pigmentepithelzellen zurückzuführen sein könnte. Gelegentlich findet man im Papillengewebe, meist in der Verlängerung der Reihe der Pigmentepithelzellen, versprengte vereinzelte oder in kleinen Verbänden liegende Pigmentepithelzellen, denen man wohl keine Bedeutung beigemessen hat. In einer gemeinsam mit Elsch nig ${ }^{60}$ ) verfaßten Arbeit sind diese Verhältnisse beschrieben und abgebildet, in einer späteren Arbeit habe ich ${ }^{61}$ ) nochmals darauf aufmerksam gemacht. Wichtig ist der Umstand, daß sich solche Epithelversprengungen bereits an embryonalen Augen nachweisen lassen, somit das Vorkommen von Drusen im jugendlichen Alter trotz ihres langsamen Wachstums begreiflich erscheint. Klinisch kann man ja auch hier und da in größerer oder geringerer Entfernung vom Papillenrande Pigmentpünktchen in der Papille finden. Es wäre denkbar, daß die Bildung von Drusen in der Papille in Beziehung zu solchen versprengten Pigmentepithelzellen zu bringen wären. Es sei darauf hingewiesen, 
daß die Drusen der Glaslamelle als Abscheidungen der Pigmentepithelien betrachtet werden. Silva ${ }^{51}$ ) hat Drusen in der Netzhaut beschrieben, die zweifellos in Beziehungen zu Pigmentepithelzellen stehen, die sich in der Netzhaut fanden. Wetzel ${ }^{50}$ ) beschreibt in seinem Falle Drusen in der Papille der Netzhaut der Umgebung und in der Pars ciliars retinae; in einem zweiten Falle fand er sie massenhaft in den äußeren Schichten der abgelösten Netzhaut. Vielleicht gehört auch der Fall von $\mathrm{Ba}_{\mathrm{a}} \mathrm{s}^{39}$ ) hierher. Aus den angeführten Tatsachen ergibt sich ein gesetzmäßiger Zusammenhang der Drusenbildung auf der Glaslamelle und in der Netzhaut mit Tätigkeit von Pigmentepithelzellen, die bei senilen oder anderweitigen degenerativen Veränderungen des Auges zur Bildung von Drusen angeregt werden. Es wäre wohl zulässig anzunehmen, daß unter gewissen Verhältnissen auch in der Papille versprengte Pigmentepithelzellen befähigt werden, Drusen abzuscheiden; diese Ansicht hätte den Vorteil, für alle derartige Vorkommnisse eine einheitliche histologische oder besser gesagt histochemische Erklärung zu bieten. Sie würde aucb die Tatsache verständlich machen, daß sich Drusen meist in der Nähe des Papillenrandes entwickeln, wo sich auch versprengt Pigmentepithelzellen am häufigsten finden. Freilich ist zuzugeben, daß die in diesem einen Fall erhobenen Befunde nicht genügen, um diese Möglichkeit zur Gewißheit zu erheben. Bei der Untersuchung ähnlicher Fälle wäre aber auf diesen Umstand zu achten. Vielleicht kommt man dann auf diesem Wege der Lösung der vollkommen ungeklärten Frage nach dem Ursprunge der Drusen im Sehnervenkopfe näher.

Die Meinungen über die Beziehungen von Drusen zu verschiedenen Augenleiden einerseits und über ihren Einfluß auf die Funktionen des Auges andererseits gehen sehr weit auseinander. Das Zusammentreffen von Drusen mit Retinitis pigmentosa (Müller ${ }^{1}$ ), Nieden ${ }^{3}$ ), Re mak ${ }^{14}$ ), Ancke ${ }^{13}$ ), Morton und Parsons ${ }^{34}$ ), To polanski $i^{45}$ )], mit Chorioretinitis [Chevallerea u ${ }^{46}$ ), Juler $\left.{ }^{57}\right)$ ], mit Stauungspapille [Streiff ${ }^{35}$ ), Hirschberg und Cirincione $\left.{ }^{20}\right)$ ], Neuritis [Stood 6 ), Sachsalber ${ }^{28}$ ), Tho mson ${ }^{30}$ ), Juler $\left.{ }^{58}\right)$ ], Sehnervenschwund [Iwa nof $f^{2}$ ), De Schweinitz ${ }^{23}$ ), Cirincione $\left.{ }^{37}\right), F e j e r^{48}$ ), Wedl und $B_{0 c k^{15}}$ ), eigene Fälle 7 und 10] hat vielfach zu der Ansicht geführt, daß die Drusen sich als Folge der genannten Erkrankungen entwickelt haben, besonders da in all diesen Fällen, ebenso wie beim Glaukom [Demaria $\left.\left.{ }^{36}\right)\right]$ der Sehnerv selbst erkrankt war. Dies führt sogar Str eiff dazu, eine Sehnervenentzündung dort anzunehmen, wo er sie nicht beweisen kann. Bei der großen Häufigkeit der Retinitis pigmentosa und der verschiedenen Arten der Chorioretinitis im Krankenmaterial aller Augenärzte ist es keineswegs verwunderlich, daß verschiedene Kombinationen von anderen Leiden mit den genannten beobachtet werden. Wenn gar jemand Gelegenheit hat, zwei Familien mit Retinitis pigmentosa und Drusen zu 
beobachten, wie dies A ncke gehabt hat, so ist es nur natürlich, daß ein Zusammenhang zwischen den zwei Zuständen gesucht wird. Nun wird zwar Retinitis pigmentosa öfters bei mehreren Mitgliedern einer Familie gefunden und auch Drusen kommen familiär vor, so daß das Zusammentreffen der beiden familiären Anlagen ein zufälliges sein könnte. Es ist aber vielleicht doch ein Zusammenhang zwischen den beiden Leiden vorhanden; da bei der Retinitis pigmentosa die Pigmentepithelien in die Netzhaut einwandern, könnte es ausnahmsweise vorkommen, daß sie in die Papille eingewandert sind und nun Anlaß zur Entstehung der Drusen gogeben haben: vielleicht hängt das $\mathrm{Zu}$ sammentreffen auch damit zusammen, daß Retinitis pigmentosa vorwiegend in Augen mit dunklem Augenhintergrund gefunden wird, in denen die Entwicklung der pigmentierten Elemente überhaupt eine reichliche ist; daß unter solchen Umständen eher eine Versprengung von Pigmentepithelien vorkommen kann, wäre erklärlich.

Wichtig ist jedenfalls das Zusammentreffen von Drusen mit primären Sehnervenerkrankungen, Sehnervenentzündung und Sehnervenschwund. In den Fällen, in denen eine Ursache für die Erkrankung des Sehnerven gefunden wurde, ist es wohl richtig die Atrophie, sei sie eine vollständige, sei sie eine teilweise, auf die Grundkrankheit zu beziehen (Hydrocephalus internus, Geschwulst der Hypophyse, Lues, Glaukom). Hier würde sich nur die Frage ergeben, ob die Drusenbildung als die Folge der Sehnervenerkrankung anzusehen ist, oder ein anderer Zusammenhang zwischen beiden Zuständen besteht. Bei dem ungemein langsamen Wachstum der Drusen, wie dies durch die Fälle von $\mathrm{Ni}$ i d e n, und meine Fälle 4, 12 und 14 belegt wird, ist kaum anzunehmen, daß eine kurzdauernde Erkrankung den Anlaß zur Drusenbildung gegeben hat. Hier ist das Zusammentreffen wohl als ein Zufalliges zu betrachten und die Annahme gerechtfertigt, daß die Drusen schon vor dem Auftreten dor Sehnervenerkrankung bestanden haben, so in den Fällen von Stauungspapille, Alkohol-Tabak Amblyopie und Neuritis. Es muß aber als möglich zugegeben werden, daß in den Fällen langsamen Sehnervenschwundes aus verschiedenen Ursachen das Verschwinden der Nervenfasern fördernd auf die Entwicklung der Drusen gewirkt hat, indem vielleicht der Gewebsdruck abgenommen hat oder beim Zerfall der Nervenfasern Stoffe frei geworden sind, die als Baumaterial für die Drusenentwicklung gedient haben. Dies sind selbstverständlich nur Mutmaßungen, die nicht bewiesen werden können. Der Sehnnervenschwund an sich genügt wohl aber nicht um den Anstoß zur Entwicklung von Drusen zu geben, wenn die Anlage nicht schon früher vorhanden war. Dafür spricht einerseits die große Zahl der unkomplizierten Fälle von Drusen im Sehnervenkopf, andererseits die Häufigkeit des Sehnervenschwundes ohne Drusenbildung. 
Findet sich eine Sehnervenerkrankung als Folge einer Geschwulst der Hypophyse oder eines Hydrocephalus internus oder eines wohl diagnostizierbaren Leidens, so ist, wie erwähnt, die Erklärung für den Sehnervenschwund gegeben und es ist überflüssig die Anwesenheit der Drusen im Sehnervenkopfe für die Erklärung des Sehnervenschwundes heranzuziehen. Anders ist dies jedoch, wenn der Sehnervenschwund ohne erkennbare Ursache bei gleichzeitiger Anwesenheit von Drusen auftritt. Es ist jedenfalls auffällig, wie häufig bei der anatomischen Untersuchung von Drusen in der Papille Sehnervenschwund festgestellt wurde ohne eine anatomische Erklärung für die Entstehung der Atrophie. Hirschberg und Cirincione ${ }^{20}$ ), Gurwitsch ${ }^{21}$ ), De Sehweinitz ${ }^{23}$ ), Cirincione ${ }^{37}$ ) und mein Fall dienen als Belege. De Schweinitz hat die Obduktion seines Falles gemeinsam mit Dercum durchgeführt; es müßte einem so erfahrenen Neurologen aufgefallen sein, wenn anatomische Veränderungen des Gehirnes vorgelegen hätten: im Obduktionsbefunde ist jedoch von diesen nicht die Rede. Sie fehlen ebenso im Falle von $\mathrm{Sachsalber}$ und im meinigen. Betrachtet man die anatomischen Abbildungen der Sehnerven bei Anwesenheit von Drusen im Sehnervenkopfe, so fällt einem auf, daß in den meisten die Drusen nicht nur in der Ebene der Netzhaut oder vor derselben zwischen den Nervenfasern liegen, sondern daß sie sich im Sklerotikochorioidealkanale befinden, ja sogar die Lamina cribrosa zurückdrängen. Hier ist es aus rein mechanischen Gründen nicht wohl denkbar, daß die Drusen einfach die Nervenfasern beiseite schieben ohne irgendeinen Einfluß auf sie auszuüben. Dies läßt sich allenfalls in den Fällen annehmen, in denen die Drusen in den oberflächlichen Schichten der Papille liegen; das Gewebe kann gegen den Glaskörper ausweichen und erleidet höchstens eine geringe Dehnung. Bei der langsamen Entwicklung der Drusen kann sich das Gewebe dieser Dehnung anpassen. Sitzen aber die Drusen im Sklerotokochorioidealkanale, so müssen sie mit der Zeit einen Druck auf die Sehnervenfasern üben, was einen Schwund dieser letzteren zur Folge haben muß. In der Tat sehen wir in den ausgesprochenen Fällen von Drusen stets einen deutlichen Schwund der Nervenfasern, den auch die Forscher beschreiben, ohne allerdings einen Grund hierfür anzugeben. Auch bei der Durchsicht einiger Schnitte des Falles von Sachsalber, die mir Herr Professor Meller in liebenswürdiger Weise zum Studium überließ, fand ich im retrolaminaren Teil des Sehnerven, der hinter den prälaminar liegenden Drusen sich befindet, eine deutliche Atrophie des Nervengewebes, die Sachsalber nicht erwähnt hat. Die von ihm beschriebene zellige Infiltration besteht auch in diesen Teilen. Also anch hier läßt sich anatomisch die Schädigung der Nervenfasern durch die Drusen erkennen.

Es ist notwendig die Funktion der unkomplizierten Fälle von Drusen 
in der Papille zu betrachten. Stood ${ }^{6}$ ) nimmt in seinen beiden Fällen das Vorhandensein einer Sehnervenentzündung. an; diese ist meines Erachtens im zweiten Falle nicht erwiesen, da die Drusen allein die unscharfe Begrenzung der Papille und ihre helle Farbe bedingen können. Auch im ersten Falle ist es nicht sicher, daß eine Neuritis bestanden hat; dieser Fall läßt sich in ähnlicher Weise deuten, wie dies auch Höegit) in seinem Falle 4 bedingt annimmt. In beiden Fällen von Stood war eine deutliche Gesichtsfeldeinschränkung vorhanden. He $\mathrm{y}^{27}$ ) erwähnt in seinem Falle eine nasale Einschränkung, ähnlich wie beim Glaukom. Morton und Parsons ${ }^{34}$ ) führen in ihrem zweiten Falle das Vorhandensein von kleinen Skotomen an. Im Falle von Waardenburg ${ }^{58}$ ) bestand eine starke Gesichtsfeldeinschränkung in der oberen Hälfte mit einem Skotom, das den blinden Fleek mit einschloß und oben mit der peripheren Grenze des Gesichtsfeldes zusammenhing. In vielen Fällen ist das Gesichtsfeld nicht untersucht worden, oder die Untersuchung wurde nur am Perimeter vorgenommen. Eine genaue Untersuchung nach Bjerrum erwähnt ausdrücklich nur Höeg.

Bei der Betrachtung der Gesichtsfelder in meinen eigenen Fällen muß darauf hingewiesen werden, daß in den ältesten Fällen $(1-3)$ die Gesichtsfeldaufnahme nur am Perimeter erfolgte: bei der Nachuntersuchung des Falles 3 wurde ebenso wie in allen anderen eine genaue Untersuchung nach Bjerrum vorgenommen. Im Falle 9 konnte aus äußeren Gründen kein Gesichtsfeld aufgenommen werden. Für die Beurteilung des Funktionsausfalles, der sich infolge der Anwesenheit von Drusen in der Papille findet, müssen die Fälle 7 and 10 gleichfalls ausscheiden, da hier im ersten Falle die Erblindung auf eine Geschwulst der Hypophyse, im zweiten die Herabsetzung der Augenfunktion auf eine Alkohol-Tabak Amblyopie zu beziehen sind. Es bleiben somit von meinen 14 Fällen nur 9 übrig, bei denen die Gesichtsfelduntersuchung verwertbar ist, da sie sorgfältig nach dem Bjerrumsehen Verfahren untersucht worden sind. In 14 Augen dieser Kranken waren Drusen vorhanden und davon wiesen 9 Augen Gesichtsfeldveränderungen auf. In einem Falle war nur eine periphere Einschränkung geringen Grades für Weiß und für Farben vorhanden; im Falle 3 wies das rechte Auge eine deutliche periphere Einschränkung für Weiß, besonders aber für Farben auf; diese Einschränkung war im schwerer betroffenen linken Auge deutlicher ausgesprochen und hier fand sich auch ein Skotom nahe dem blinden Fleck. Im Falle 14 hatte schon die erste Untersuchung im Jahre 1904 Ausfälle des Gesichtsfeldes ergeben, die sich im Laufe der Zeit mit der Zunahme der Drusen und der Opticusatrophie steigerten. In drei Fällen $(6,8,13)$ mit 6 beteiligten Augen fanden sich fünfmal kleine Gesichtsfeldausfälle in der Nähe des Fixationspunktes oder des 
blinden Fleckes. Es ist nicht ausgeschlossen, daß auch in anderen Fällen weiter peripher liegende kleine Skotome vorhanden waren, deren Nachweis aber nicht gelingen konnte.

Zieht man nun die Häufigkeit der Ausfälle im Gesichtsfelde bei genauer Untersuchung in Betracht, so muß man zum Schlusse kommen, da diese mit der Anwesenheit der Drusen in Zusammenhang stehen, d. h. da $B$ die Drusen auch bei geringer Ausbildung imstande sind, kleinere Gruppen von Sehnervenfasern zu schädigen und dadurch einen Ausfall im Gesichtsfeld hervorzurufen. Sind die Drusen in größeren Masson anwesend, so führen sie zu schwerer Schädigung der Sehnervenfasern; diese gehen mit der Wachstumszunahme der Drusen parallel: es entstehen größere Gesichtsfeldausfälle, leiden die makularen Fasern, so sinkt auch die zentrale Sehschärfe. In seinem zweiten Falle macht Höeg den Stiel der in den Glaskörper hineinragenden Drusenmasse für den vorhandenen Gesichtsfelddefekt verantwortlich. Nettleshi $\mathrm{p}^{16}$ ) hat in einer Aussprache zu einer Demonstration von Werner über Chorioiditis guttata centralis der Meinung Ausdruck gegeben, da. dabei vielleicht in der Tiefe der Papille liegende, mit dem Augenspiegel nicht sichtbare Drusen am Funktionsausfalle der Augen Schuld seien. Ist die Drusenmasse so groß wie in meinem Falle, so ist es klar, daß es zu einem hochgradigen Sehnervensehwunde kommen muß. Man kann an der Hand der beschriebenen Fälle alle Zwischenstufen zwischen vollständig normaler Funktion der Augen und vollständigem oder nahezu vollständigem Sehnervenschwunde feststellen in Fällen, bei denen andere Schädlichkeiten, als die Drusen, nicht in Betracht kommen. Es unterliegt keinem Zweifel, daß im Beginn ihrer Entwicklung die Drusen das Nervengewebe nicht $z u$ schädigen brauchen, und daß in vielen Fällen die Schädigung des Auges eine so geringe ist, daß sie nur bei der gegenauesten Untersuchung nachgewiesen werden kann. Daher ist es begreiflich, wenn Köhler ${ }^{56}$ ) unter 8 Fällen keinen Ausfall an Funktion erwähnt. Eine Gesichtsfelduntersuchung nach Bjerrum scheint in seinen Fällen nicht vorgenommen worden zu sein. In einer ähnlichen Lage war ich bei meinen ersten 4 Fällen. Erst als ich daran ging, alle Fälle genau zu untersuchen, kam ich dazu, die Häufigkeit kleiner Gesichtsfeldausfälle zu erkennen und damit brach die Ansicht bei mir Bahn, daßs die Drusen keine ganz unschuldigen Bildungen sind, als welche sie bisher aufgefaßt wurden. Diese Beobachtungen führten zu einer andern Ansicht über die Beziehungen zwischen Funktionsausfall und Drusenbildung, als die bisher angenommene. Die klinische und anatomische Untersuchung des durch 10 Jahre beobachteten Falles, den Rabitsch zuerst veröffentlicht hatte, drängt nun vollends zur Ansicht, daß hier der Sehnervensehwund durch die massenhafte Drusenbildung hervorgerufen worden war. Die kritische Durchsicht der veröffentlichten 
Fälle kann diese Ansicht nur bestätigen. Die zeitweisen Remissionen der Störungen des Sehvermögens, die schon Nieden wahrgenommen hatte, und die im vorliegenden Falle gleichfalls aufgetreten sind, sind schwer zu erklären, da eine Rückbildung der Drusen nicht angenommen werden kann; es kann sich eher um eine andere unbekannte Ursache handeln, die eine vorübergehende Verschlechterung des Zustandes hervorgerufen hatte.

Es muß also festgestellt werden, daß es eine teilweise Atrophie des Sehnerven gibt, die auf die Anwesenheit von Drusen im Sehnervenkopfe zurückzuführen ist und die mitunter zu schweren Sehstörungen Anlaß geben kann.

Wenn die vorstehenden Ausführungen geeignet sind eine richtigere Ansicht über die klinische Bedeutung der Drusen im Sehnervenkopfe zu begründen, so sind sie leider nicht genügend um eine Sicher heit bezüglich der Ursache der Drusenbildung zu geben. Die anatomische Untersuchung und die Analogie mit andern Drusenbildungen lassen die Möglichkeit als berechtigt erscheinen, daß die Drusen auf versprengte Pigmentepithelzellen zurückzuführen sind. Dies würde es auch verständlich erscheinen lassen, daß die Drusen immer nur im prälaminaren Teile des Sehnerven auftreten, dann nur hier können sich versprengte Pigmentepithelzellen finden. Weitere Untersuchungen auf diesem Gebiete werden uns vielleicht Aufklärung darüber bringen.

\section{Literaturverzeichnis.}

1) Müller, H., Über Niveauveränderungen an der Einstrittsstelle des Sehnerven. Arch. f. Ophthalmol. 4, $2,1.1858 .-{ }^{2}$ ) I wa noff, Über Neuritis optica. Klin. Monatsbl. f. Augenheilk. 6, 421. 1858. - ") Niede n, A., Über Massenentwicklung von Drusen der Lamina vitrea chorioideae, nur im Umfange des intraokularen. Sehnervenendes. Centralbl. f. prakt. Augenheilk, 2, 6. 1878. 4) Oeller, J., Beiträge zur Lehre von der Chorioretinitis pigmentosa. Arch. f. Augenheilk. 8, 435. 1879. - ${ }^{5}$ ) Jany, Zur Kasuistik der Drusenbildung in der Lamina vitrea chorioideae an der Papilla nervi optici. Centralbl. f. prakt. Augen. heilk. 3, 167. 1879. - ${ }^{6}$ ) Stood, Über zwei Fälle von Drusenbildung am intraw okularen Sehnervenende. Klin. Monatsbl. f. Augenheilk. 21, 506. 1883. - 7) Lawson, G., Syphilitic chorioretinitis with pesuliar growths at the fundus. Trans. Ophth. Sore. of the Unit. Kingd. 3, 117. 1883. ${ }^{8}$ ) Story, J. B., Anomalous distribution of retinal arteries. Tbidem 3, 102. 1883. - ${ }^{9}$ ) Masselon, Infiltration vitreuse de la rétine et de la papille. Paris 1884. - ${ }^{10}$ ) Sehaefer, Anatomische Beschreibung eines Auges mit Iridochorioiditis suppurativa. Centralbl. f. prakt. Augenheilk. 8, 203. 1884. - ${ }^{11}$ ) Hirschberg, J., Notiz zum Vortrag Masselon in der Soc. Ophth. de Paris. Ibidem 8, 46. 1884. - ${ }^{12}$ ) Hirschberg und Birn. bacher, Beiträge zur Pathologie des Sehorgans, VI. Ibidem 9, 65. 1885. 13) Ancke, R., Beiträge zur Kenntnis von der Retinitis pigmentosa. Ibidem 9, 167. 1885. - 14) Re mak, Ein Fall von exzessiver Drusenbildung in der Papille bei atypischer Retinitis pigmentosa. Ibidem 9, 257. 1885. - ${ }^{15}$ ) Wedl und Bock, Pathologische Anatomie des Auges. 1886, S. 229. - ${ }^{16}$ ) Nettleship, Aussprache 
zu: Werner, Vitreous infiltration of the retina and central guttate chorioiditis. Trans. Ophth. Soc. of the Unit. Kingd. 6, 358. 1886. - $\left.{ }^{17}\right)$ Rath, W., Geschwülste der Hypophysis cerebri. Arch. f. Ophthalmol. 34, 4, 81. 1888. - ${ }^{18}$ ) Gess ner, C., Enophthalmus traumaticus. Arch. f. Augenheilk. 18, 297. 1888. - ${ }^{19}$ ) Nieden, A., Über Drusenbildung in und um den Opticus. Arch. f. Augenheilk. 20, 72. 1889. ${ }^{20}$ ) Hirschberg, J. and Cirincione, C., Über Drusen im Sehnervenkopf. Centralbl. f. prakt. Augenheilk. 15, 166. 1891. - ${ }^{21}$ ) Gurwitsch, M., Über hyaline Bildungen im Sehnervenkopfe und in der Netzhaut bei Morbus Brightii. Ibidem 15, 225. 1891. - 22) Purtscher, O., Drusenbildung im Sehnervenkopf. Ibidem 15, 292. 1891. - ${ }^{23}$ ) De Schweinitz, G. E., Hyaline bodies (Drusen) in the nervehead Trans. Americ. Ophthalmol. Soc. 6, 349. 1892. - ${ }^{24}$ ) Terson, A., Les verrucosités hyalines de la portion papillaire du nerf optique. Arch. d'Ophth. 12, 367. 1892. - ${ }^{25}$ ) De $8 \mathrm{ehweinitz,} \mathrm{G.} \mathrm{E.,} \mathrm{Colloid} \mathrm{disease} \mathrm{in} \mathrm{the} \mathrm{macular}$ region analogous in appearance to the so-called drusen in the nerve-head. Trans. Americ. Ophth. Soc. $\gamma$, 212. 1894. - ${ }^{26}$ ) Peters, Ein Fall von Drusenbildung im Sehnerven. Allg. Zeitschr. f. Psych. 50, 5, 1093. 1894. - ${ }^{27}$ ) Heyl, Albuninoid(?) deposit in the optic dise and retina. Trans. Americ. Ophth. Soc. $\gamma, 355.1895$. $\left.{ }^{28}\right)$ Sachsalber, A., Beitrag zur Drusenbilaung im Sehnervenkopfe. Beitr. zur Augenheilk. 5, 1. 1895. -- ${ }^{29}$ ) Gifford, H., An unusual case of hyaline bodies in the optic nerve. Arch. of Ophth. 24, Nr. 3. 1895. - ${ }^{30}$ ) Thomson, E. S., Colloide degeration of the optie nerve. The Postgraduate, New York. 1898. - ${ }^{31}$ ) Streiff, Über Altersveränderungen der Vasa centralia retinae nebst einem Beitrag zur Kenntnis der Konkremente des Opticus und seiner Scheiden. Inaug.-Diss. Zürich 1898. - ${ }^{32}$ ) Nieden, A., Drusenbildung im Opticus. Vers. rheinisch-westf. Ärzte in Bochum. Zeitschr. f. Augenheilk. 3, 361. 1900. - ${ }^{33}$ ) Mendel, F., Über Drusenbildung im Sehnervenkopfe. Zeitschr. f. prakt. Augenheilk. 24, 242. 1900. ${ }^{34}$ ) Morton. A. S. und Parsons, J. H., Hyaline bodies (Drusenbildungen) at the optic disc. Trans. Ophth. Soc. Unit. Kingd. 23, 135, 1903. - ${ }^{35}$ ) Streiff, J. J., Über die Entstehung der Opticusdrusen. Klin. Monatsbl. f. Augenheilk. 42, 1, 149. 1904. - ${ }^{36}$ ) Demaria, B., Zur Pathogenese der Amotio chorioideae nach Iridektomie bei Glaukom und über Corpora amylacea in der exkavierten Papille. Ibidem 42, 1, 339. 1904. - ${ }^{37}$ ) Cirincione, C., Concrezioni nella testa del nervo ottico. La clin. oculis, 1904. - ${ }^{38}$ ) Hanke, V., Ein Fall von hyaliner Excrescenz der Chorioidea. Ophth. Ges. in Wien. Zeitschr. f. Augenheilk. 11, 86. 1904. ${ }^{39}$ ) Baas, K. Krystalldrusen in und unter der abgelösten Netzhaut. Arch. f. Ophth. 57, 571. 1904. - ${ }^{40}$ ) Harman, N., B., Hyaline degeneration of the optic disc. Trans. Ophth. Soc. Unit. Kingd. 14. 12. 1905. - ${ }^{11}$ ) Rabitsch, F., Zur Kenntnis der Drusen im Sehnervenkopf. Klin. Monatsbl. f. Augenheilk. 43, 72. 1905. - ${ }^{42}$ ) Schuster, Zur Kasuistik krystallähnlicher Gebilde des Auges. II. Ein Fall von Drusenbildung im. Sehnervenkopf. Arch. f. Augenheilk. 54, S. 364. 1906. 43) Bonhoff, Bericht über die Wirksamkeit der Universitäts-Augenklinik zu Gießen. Inaug.-Diss. Gießen 1906. - ${ }^{44}$ ) La uber, H., Drusen in der Papille. Ophth. Ges. in Wien. Zeitschr. f. Augenheilk. 17, 391. 1907. - ${ }^{45}$ ) To polanski, A. Drusen am Sehnerveneintritt und Pigmentdegeneration der Retina. Ophth. Ges. in Wien. Tbidem 20, 584. 1908. - ${ }^{46}$ ) Chevallereau, Verrucosités hyalines du nerf optique et de la chorioide. Soc. Ophth. de Paris. Recueil d'Opht. 31, 103. 1909. - ${ }^{47}$ ) Höeg, N., Über Drusen im Sehnervenkopf. Arch f. Ophthalmol. 69, 355. 1909. - ${ }^{48}$ ) Fejer, Über Drusen des Sehnervenkopfes. Arch. f. Ophthalmol. 29, 454. 1909. - 49) Kärnbach, Über Drusenbildung im Sehnervenkop£ des Pferdes. Monatssehr. f. prakt. Tierheilk. 21, 481. 1910, - a0) Wetzel, E., Über Drusen im Sehnervenkopf und in der Retina. Inaug.-Diss. Rostock 1911. 51) Silva, R., UUber Drusen der Chorioidea und der Retina. Klin. Monatsbl. f. 
Augenheilk. 49, 379. 1911. - ${ }^{52}$ ) La uber, H., Drusen des Sehnerven, Ophth. Ges. in Wien. Zeitschr. f. Augenheilk. 29, 201. 1913. - ${ }^{53}$ ) Coats, G., Concretions in the papilla and corpora amylacea in the retina. Trans. Ophth. Soc. Unit. Kingd. 32, 119. 1912. - ${ }^{54}$ ) Wilbrand und Saenger, Neurologie des Auges 4, 495. 1912. - - ${ }^{5}$ ) Paderstein, Drusen des rechten Sehnervenkopfes. Centralbl. f. prakt. Augenheilk. 36, 18. 1912. - ${ }^{56}$ ) Köhler, H., Zur Kasuistik der Drusen im Sehnervenkopfe. Inaug.-Diss. GieBen 1913. - ${ }^{57}$ ) Juler, Hyaline bodies of the optic disc. Trans. Ophth. Soc. Unit. Kingd. 34, 177. 1914. - ${ }^{58}$ ) Waarden. burg, S. P., Krystalloide vormsels in het netvlies gepaard met een eigenaardige gezichtsveldstoornis. Nederl. Tijdschr. v. Geneesk. II, S. 690. 1917. (Ref. Klin. Monatsbl. f. Augenheilk. 59, 690. 1917.) - 59) Stargardt, Über Drusen am Sehnervenkopf. Niederrh. Ges. f. Natur- und Heilk. Deutsche med. Wochensch?. Nr. 49, S. 1374. 1919. - $\left.{ }^{60}\right)$ Elschnig und Lauber, Über die sog. Klumpenzellen der Iris. Arch. f. Ophth. 65, 428 (S. 435 und Taf. XIX, Abb. 5). 1907.-61) La uber, H., Beiträge zur Entwicklungsgeschichte und Anatomie der Iris und des Pigmentepithels der Netzhaut. Areh. f. Ophthalmol. 68, 1. 1908. 